Mitteilungen der Österreichischen Geographischen Gesellschaft, 160. Jg., S. 163-176

(Annals of the Austrian Geographical Society, Vol. 160, pp. 163-176)

Wien (Vienna) 2018, https://doi.org/10.1553/moegg160s163

\title{
Migration
}

MIGRATION

\section{Tibetische Migrantenökonomie in Majnu Ka Tilla, DELHI / INDIEN}

\author{
Jan-Christoph ScHLENK, Bonn, und Frauke KrAAS, Köln* \\ Erste Einreichung / Initial submission: 04/18; revidierte Fassung / revised submission: 10/2018, \\ endgültige Annahme / final acceptance: 11/2018 \\ mit 5 Abb. im Text
}

INHALT

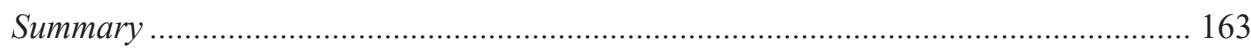

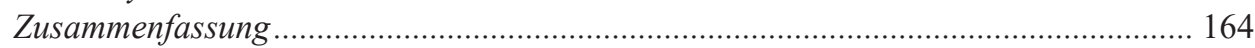

1 Ethnische Ökonomien tibetischer Migranten in Indien........................................... 165

2 Die Entwicklung des Stadtviertels Majnu Ka Tilla................................................ 166

3 Migrantenökonomie im tibetischen Viertel............................................................ 167

4 Entwicklungspfade im tibetischen Viertel - Zukunft und Potenziale ökonomischer

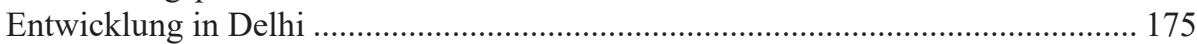

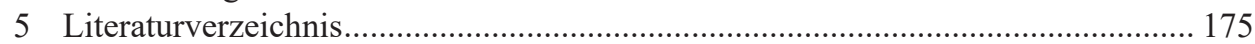

\section{Summary}

\section{Tibetan Immigrant Economy in Majnu Ka Tilla, Delhi / India}

The article focuses on the immigrant economy of the Tibetan exile community in India's capital by examining the development of the settlement Majnu Ka Tilla at the flood plains of the Yamuna River. Majnu Ka Tilla developed from a squatter settlement

\footnotetext{
* Dipl.-Geogr. Jan-Christoph SchlEnK, Berater im Sektorvorhaben Nachhaltige Sanitärversorgung, Deutsche Gesellschaft für Internationale Zusammenarbeit (GIZ) GmbH, Friedrich-Ebert-Allee 36, D-53113 Bonn, Deutschland; Univ.-Prof. Dr. Frauke KraAs, Geographisches Institut, Universität zu Köln, Albertus-Magnus-Platz, D-50923 Köln, Deutschland; E-Mail: jan.schlenk@giz.de, f.kraas@uni-koeln.de,www.geographie. uni-koeln.de
} 
in the 1960s into the Indian centre of Tibetan commerce in exile and a tourist hotspot. The economic development potential of Majnu Ka Tilla - within the legal framework of Delhi - is investigated by analysing the data of a qualitative and quantitative field study grounded in concepts of ethnic economies. The collected data show that the first migrants started with very simple businesses like food stalls, small pubs, brewing of Tibetan beer, and small-scale textile enterprises - mostly 'sweater-selling'-which enabled them to survive in the city. The second generation, better educated than their parents, was already able to open stores allowing them to achieve greater financial independence. With increased options, economic activities became more diverse as demonstrated by the opening of travel agencies, beauty parlors and hotels. The 'microcosm' of their own ethnic businesses forms the foundation for integration into urban society, a further economic diversification based on better education and the establishment of international economic networks.

Keywords: Immigrant economy, ethnic economy, Tibetan migrants, informal settlement, Delhi, India

\section{Zusammenfassung}

Der Artikel behandelt die Untersuchung der Migrantenökonomie der tibetischen Exilgemeinde in der indischen Hauptstadt Delhi. Der Fokus liegt auf der Entwicklung der Siedlung Majnu Ka Tilla in der Flussaue der Yamuna. Die Siedlung hat sich seit den 1960 er Jahren von einer informellen Siedlung zum indischen Zentrum für den Handel mit tibetischen Waren und zu einem attraktiven Ort für den Tourismus entwickelt. Mit Hilfe qualitativer und quantitativer Erhebungen auf der konzeptionellen Grundlage ethnischer Ökonomie wurden die wirtschaftlichen Entwicklungspotenziale von Majnu Ka Tilla innerhalb der rechtlichen Rahmenbedingungen der Stadt Delhi untersucht. Die Ergebnisse zeigen, dass die erste Generation der Migranten mit einfachen Verkaufsständen für Lebensmittel, kleinen Lokalen, dem Brauen tibetischen Biers und mit Kleinbetrieben im Bereich des Textilhandels begannen, um in der Stadt überleben zu können. Die zweite Generation, besser ausgebildet als ihre Eltern, eröffnete eigene Geschäfte, um mehr ökonomische Unabhängigkeit zu erreichen. Mit erweiterten Optionen entwickelte sich eine zunehmend größere Vielfalt an wirtschaftlichen Aktivitäten - dazu zählen Reisebüros, Schönheitssalons und Hotels. Der „,Mikrokosmos “ eigener ethnischer Unternehmen bildet nun die Grundlage für eine Integration in die urbane Gesellschaft, für eine weiterhin zunehmende ökonomische Diversifizierung basierend auf höherer Bildung - und mittlerweile sogar für die Etablierung internationaler ökonomischer Netzwerke.

Schlagwörter: Ethnische Ökonomie, Migrantenökonomie, Tibetische Migranten, Informelle Siedlung, Delhi, Indien 
Dharamsala is considered the heart of the Tibet world. But it is Majnu Ka Tilla that constitutes the commercial centre of the exile community. It is the hub of Tibetan commerce and spreads its limited prosperity along its many spokes to other Tibetan communities in all four directions of the subcontinent and beyond.

(SAMPHEL 2006)

\section{Ethnische Ökonomien tibetischer Migranten in Indien}

Im Rahmen zunehmend internationalisierter Urbanisierungsprozesse wird der Frage der Beteiligung migrantischer bzw. „ethnischer“ Ökonomien, ihrer kulturell-ethnischen Werte und Normen sowie sog. „ethnischer Ressourcen“ (wie etwa Gründungskapital oder Zuliefer- und Handelsnetzwerke) zunehmend Beachtung geschenkt (vgl. grundlegend dazu: Kulinna 2007; Tolciu und Schaland 2008; Pütz 2003, 2004). Diskutiert wird, ob eher traditionelle, kulturell begründete Geschäftspraktiken (etwa: orientalische Handelstraditionen) wirken oder ob sich Selbstständigkeit in Reaktion auf Strukturwandel durch Akzeptanz schlechter Arbeitsbedingungen und geringer Rentabilität primär als ein Wechsel vom „marginalen“ Arbeiter zum „marginalen“ Unternehmer vollzieht - als Teil einer Überlebensstrategie angesichts mangelnder beruflicher Alternativen und in Reaktion auf überdurchschnittlich steigende Arbeitslosigkeit.

Die Selbstständigkeit von Migranten als Opportunität oder Notwendigkeit wird in der Regel durch die Länge der Aufenthaltsdauer, übergeordnetes wirtschaftliches Interesse oder lokale Möglichkeiten begünstigt. Gründungen entstehen aber auch im Zusammenhang mit „Nischenökonomien“ durch Nachfrage nach speziellen Konsumgütern (LiGHT und RoSENSTEIN 1995; SCHALAND 2009). In diesem Zusammenhang stellt sich auch die Frage, inwiefern Selbstständigkeit zur Integration beiträgt (sozialer Aufstieg oder Rückzug auf die eigene ethnische Gruppe: Fischer-KrapoHL 2010; Hillmann 2001; SCHALAND 2009).

Im vorliegenden Beitrag werden die ethnischen Ökonomien der tibetischen Flüchtlinge und Migranten, deren unternehmerische Tätigkeit als Migrantenökonomie ein Teilsegment urbaner Ökonomien in Indien darstellt, untersucht.

Anfang der 1960er Jahre, als tibetische Flüchtlinge dem Dalai Lama, ihrem politischen und geistlichen Oberhaupt, in das Exil nach Indien folgten, suchten viele von ihnen Zuflucht in indischen Städten. Der erste indische Premierminister Jawaharlal Nehru war es, der den Dalai Lama 1959 als Gast willkommen hieß und ihm gestattete, in Indien eine Exilregierung zu gründen. Allerdings machte er ihm, um die Beziehungen Indiens zu China nicht noch weiter zu gefährden, zugleich deutlich, dass Indien seine politischen Zielsetzungen nicht unterstützen würde. Als einer der Gründe für die materielle und humanitäre Hilfe Indiens wird oft die auf dem Buddhismus gründende Freundschaft zu den Tibetern angegeben (MethFessel 1995, S. 44). In diesem Kontext sind auch die Duldung der tibetischen Flüchtlinge sowie die Entstehung der tibetischen Siedlungen und Viertel in Indien zu sehen. 
Die politische Brisanz der ,Tibet-Problematik“ ist eng mit den politischen und rechtlichen Rahmenbedingungen in Indien verknüpft. Im Unterschied zu anderen Migranten erlaubt die indische Regierung den Tibetern die über ganz Indien verteilte Einrichtung eigener Stadtviertel und Siedlungen - zum Beispiel in Nordindien in Dharamsala (dem Sitz der Exilregierung), in Majnu Ka Tilla in Delhi sowie in Südindien im Bundesstaat Karnataka, mit einer der bekanntesten Siedlungen: Bylakuppe. Diese Siedlungen wurden der tibetischen Exilregierung mit dem Dalai Lama als Oberhaupt zur Verwaltung übergeben. Dadurch wurden Enklaven gesellschaftlicher Autonomie eingerichtet, so wie sie sich die tibetische Führung für den Erhalt ihrer Kultur wünschte (GoldSTEIN 1975, S. 13). In den Siedlungen wurden neben dem Aufbau von Verwaltungseinrichtungen, Schulen, Klöstern und Krankenstationen auch Geschäfte, Restaurants, Hotels und Reisebüros, zunächst für den eigenen Bedarf, eröffnet.

\section{Die Entwicklung des Stadtviertels Majnu Ka Tilla}

New Aruna Nagar Colony lautet der offizielle indische Name für das tibetische Viertel in der indischen Hauptstadt. Diesen Namen erhielt es im Jahr 2000 von Delhi's Chief Minister, Sheila Dikshit. Der Dalai Lama gab dem Viertel den tibetischen Namen Samyeling. Es gehört zum Delhi North District Assembly „, Chandni Chowk 020“. Grund und Boden sind „government land“. Dank der guten Beziehungen des Dalai Lama zu indischen Politikern konnte der im Green Yamuna Action Plan beschlossene Abriss der als ,, unauthorised colony “ eingestuften Siedlung im Jahr 2006 nach der Intervention indischer Politiker gestoppt werden. Es folgten jahrelange gerichtliche Auseinandersetzungen, die mit der Legalisierung von Majnu Ka Tilla im September 2012 endeten (Central Tibetan Administration 2012).

Im Jahr 2002 waren 60 Prozent, fünf Jahre später 2007 bereits 76 Prozent der zugewiesenen Fläche bebaut (Municipal Cooperation of Delhi 2012). Das tibetische Viertel liegt im ward (Distrikt) 78 Majnu Ka Tilla, benannt nach dem im Süden des wards liegenden gleichnamigen Sikh-Tempel. Da sich in Delhi Namen von ,,landmarks “ im Alltag der Bevölkerung und Medien oft durchsetzen, ist das tibetische Viertel in Delhi nur als Majnu Ka Tilla bekannt.

Die Siedlung befindet sich im Norden Delhis, fünf Kilometer nördlich der tibetischladakhischen Siedlung Ladakh Buddha Vihar; im Osten begrenzt die Flussaue der Yamuna das Viertel, im Westen eine mehrspurige Zubringerstraße. Das Old Camp am Sikh-Tempel ist der älteste Teil der Siedlung, nördlich schließt sich das New Camp an (Abb. 1); Zugang erhält man durch sogenannte Gates. Die einfachen, mit Wellblech gedeckten Hütten des Old Camp liegen tiefer und sind in der Monsunzeit am stärksten von Überschwemmungen betroffen (zuletzt im Herbst 2010). In den zwölf Blöcken des Viertels leben etwa 350 Familien permanent (eigenes Interview).

Als die Flüchtlingsströme ab dem Jahr 1980 im Zuge wachsender Verfolgung und Diskriminierung in Tibet wieder zunahmen und zeitgleich Häuser von Majnu Ka Tilla der Verbreiterung der Zubringerstraße weichen mussten, wurde das New Camp errichtet. Mit dieser Erweiterung und der damit verbundenen Eröffnung von Hotels, Restaurants und 

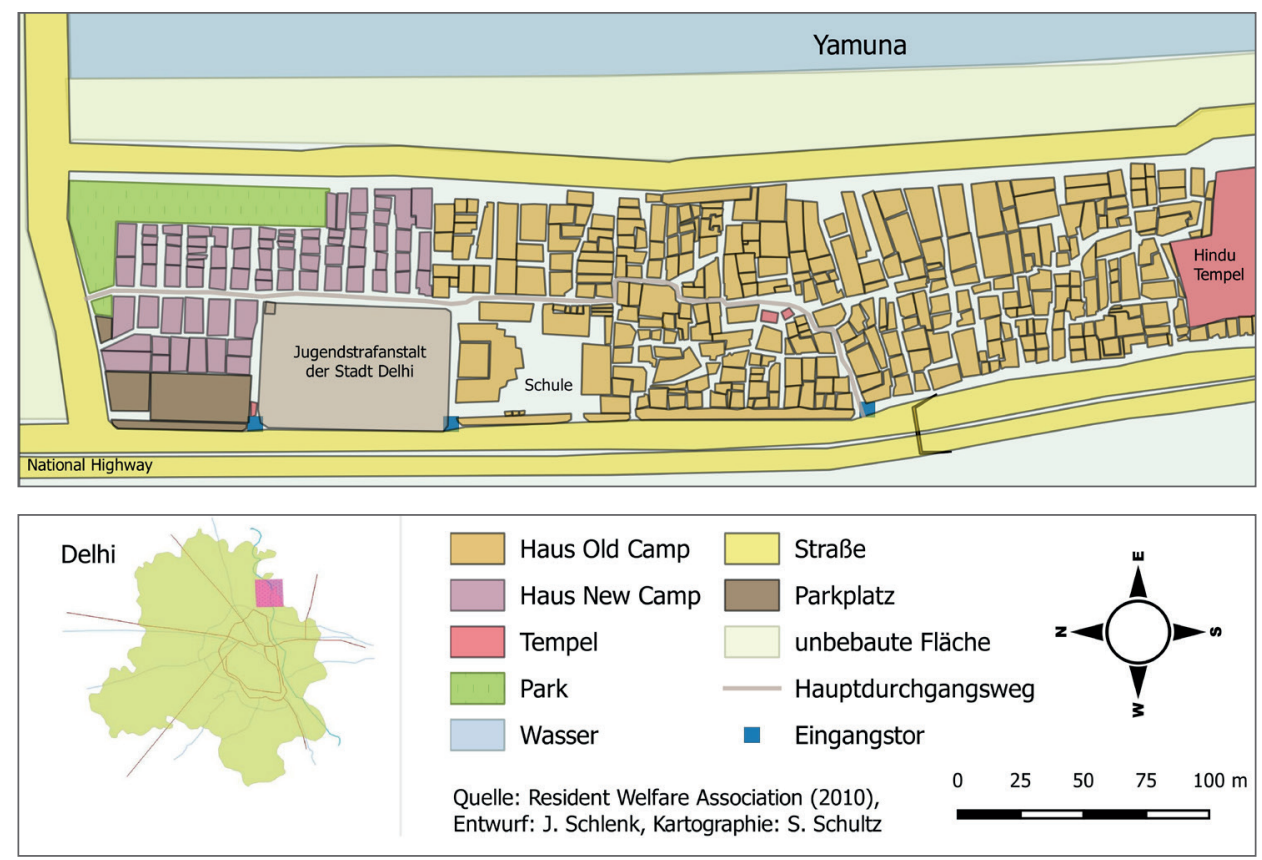

Abb. 1: Karte von Majnu Ka Tilla mit seinem Old Camp und dem New Camp

Geschäften verbesserte sich der Lebensstandard merklich. Anfang der 1990er Jahre lebten etwa 1300 Tibeter permanent in Majnu Ka Tilla. Diese Zahl verdoppelt sich während der Haupthandelssaison im Winter (MethFEssel 1995, S. 82). Ursache für diese temporäre Bevölkerungszunahme sind die saisonalen Märkte in Delhi, auf denen vor allem günstige Textilien von den sogenannten ,sweater sellers “ angeboten werden. Seit den 1990er Jahren entwickelte sich Majnu Ka Tilla immer mehr zu einem beliebten Touristenviertel, das mit seinem besonderen Flair nicht nur Tibeter und Inder anzieht, sondern auch ,westliche“ Touristen. Dies verleiht der tibetischen Migrantenökonomie wichtige ökonomische Impulse (GUPTA 2011).

\section{Migrantenökonomie im tibetischen Viertel}

Vor dem Hintergrund der skizzierten Entwicklung geht der vorliegende Beitrag auf Basis eines Forschungsprojekts ${ }^{1)}$ der Frage der aktuellen Situation der tibetischen Migrantenökonomie in Majnu Ka Tilla und ihren sozio-ökonomischen Entwicklungspotenzialen nach. In zwei empirischen Feldforschungskampagnen wurden in den Jahren 2011 und 2012 insgesamt 101 quantitative Unternehmensbefragungen und neun leitfadengestützte

\footnotetext{
1) Ausdrücklicher Dank gebührt dem Deutschen Akademischen Austauschdienst (DAAD) für die finanzielle Unterstützung von zwei Feldforschungsaufenthalten in den Jahren 2011 und 2012.
} 
Interviews mit tibetischen Unternehmern aus der ersten und zweiten Generation durchgeführt. Dabei wurden zentrale wirtschaftliche Parameter der Migrantenökonomie - vor allem die Branchenstruktur, Eigentumsverhältnisse, Kapitaleinsatz und der rechtliche Status - erhoben und zwecks qualitativer Validierung biographische Interviews ausgewertet.

\section{Allgemeine Situation}

Entlang der schmalen Gassen Majnu Ka Tillas liegen vielfältige Geschäfte, ergänzt durch ambulanten Straßenhandel. Neben Souvenirartikeln (z. B. Bildern des Dalai Lama, Gebetsfahnen) werden einfacher, günstiger Silberschmuck, Decken, Schals und Gewürze verkauft. In der Nähe von Gate No 1 liegt der zentrale Tempelplatz, der den Tibetern als Versammlungsplatz dient. In Richtung New Camp treten immer mehr restaurierte Hotels, Restaurants und Geschäfte sowie mehrgeschossige Neubauten hinzu. Im äußersten Norden des New Camp entstand in den Jahren 2002/2003 eine kleine mehrstöckige Einkaufspassage nach ,westlichem“ Vorbild. Modernisierungsprozesse erfassen inzwischen auch das Old Camp (z. B. die 2012 eröffnete Lotus Laundry). Im Osten grenzt ein Pfad die Häuser von kleinen Feldern in der Flussaue ab. Hier leben in improvisierten Hütten indische Migranten, die Gemüse - hauptsächlich das bei den Tibetern beliebte Pak Choi - anpflanzen und im tibetischen Viertel verkaufen. Die Hotels und Restaurants im Osten haben zur Flussseite die Fenster geschlossen und verhängt, um den Touristen den Geruch und den wenig schönen Anblick des Flusses zu ersparen.

\section{Unternehmensstruktur}

Den größten Anteil der Unternehmen in Majnu Ka Tilla umfasst der Einzelhandel (28 Geschäfte). Es handelt sich um Kolonialwarenläden mit breiter Produktpalette vor allem tibetischer Lebensmittel, z. B. Gewürze der tibetischen Küche und spezielle Pasta für die traditionellen Nudelsuppen der Tibeter. Einige Geschäfte bieten auch Souvenirs an. Weitere größere Gruppen bilden Reisebüros und Hotels (jeweils 17 Einrichtungen), gefolgt von Restaurants (mit vorwiegend tibetischer Küche). Es folgen Bekleidungsgeschäfte (5 Geschäfte), Buchhandlungen (4 Geschäfte), ein DVD-Shop, ein Juwelier und eine Apotheke. Außerdem arbeiten verschiedene Dienstleister im Viertel, darunter ein Arzt, ein Schneider sowie ein Import-Export-Logistiker (insgesamt 10 Unternehmen). Sieben Geschäftsleute verkaufen ihre Produkte auf der Straße (Abb. 2).

Vergleicht man die Branchenzugehörigkeit der befragten tibetischen Selbstständigen der ersten Generation, die sich ab 1960 in Majnu Ka Tilla niedergelassen haben, so wird deutlich, dass damals Einzelhandel und Restaurants dominierten und auch der Straßenverkauf, für den weniger Qualifikation und Startkapital benötigt wird, stärker war. Bei der zweiten Generation ist die Zahl der Reisebüros und Hotels gestiegen, für die mehr Kapital und umfangreichere Kenntnisse benötigt werden (Abb. 3). Auch sind mit Ärzten und Apothekern Berufe vertreten, für die der ersten Generation die Schul- und Hochschulbildung fehlte. 


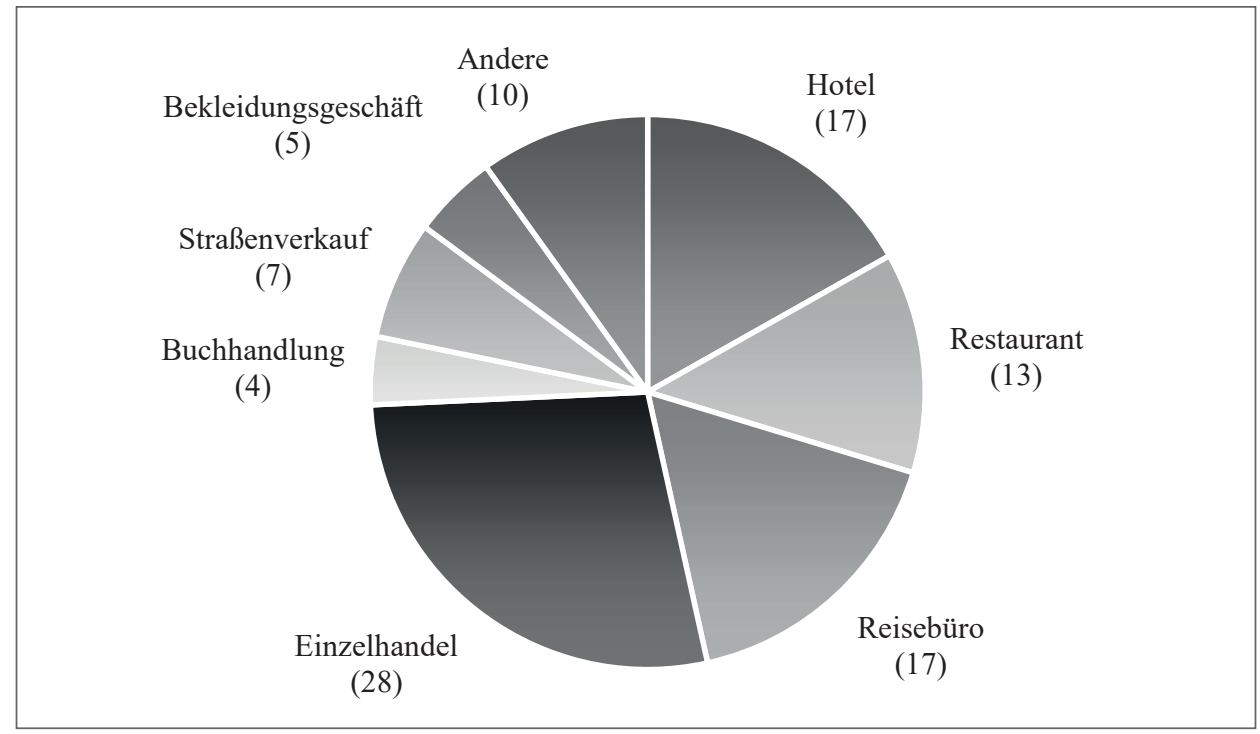

Quelle: Eigene Erhebung

Abb. 2: Branchenstruktur der tibetischen Selbstständigen in Majnu Ka Tilla ( $N=101)$

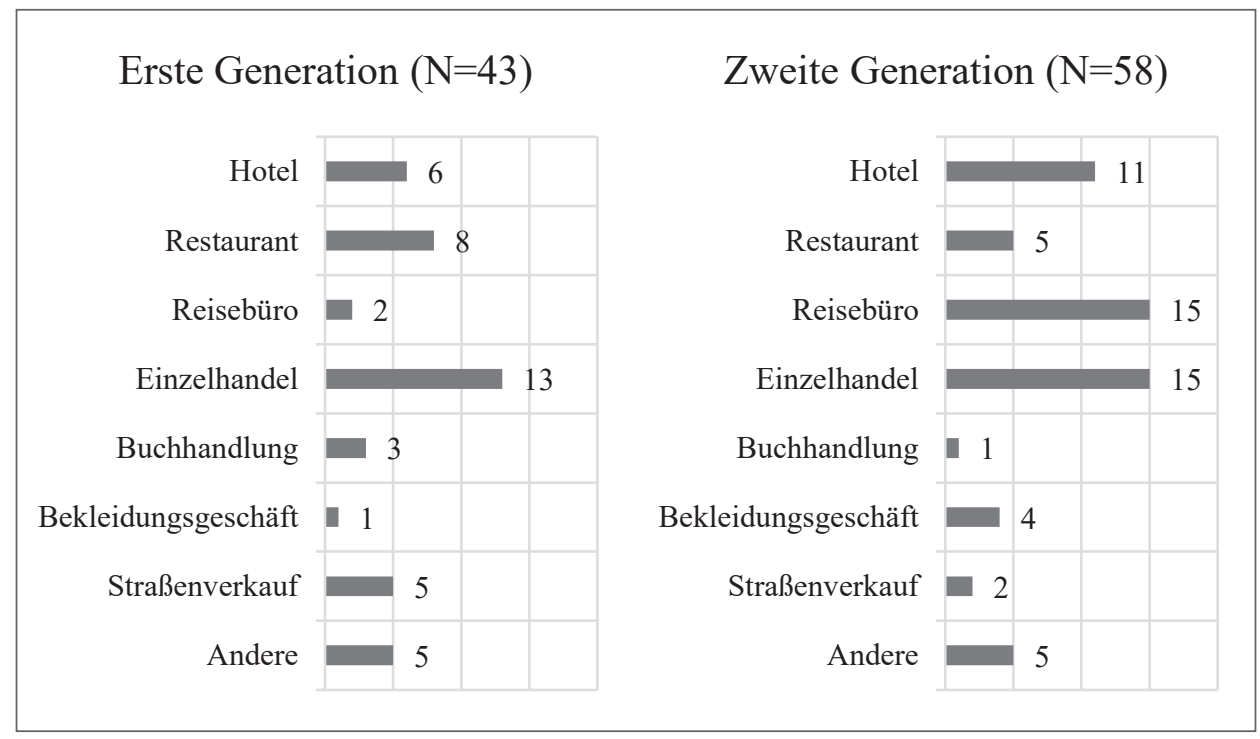

Quelle: Eigene Erhebung

Abb. 3: Vergleich der Branchenstruktur der ersten und zweiten Generation der tibetischen Selbstständigen in Majnu Ka Tilla 


\section{Entstehung und Entwicklung der Migrantenökonomie}

Während die Migranten der ersten Generation vornehmlich aus der Not heraus im Gastland kleine Wirtschaftsbetriebe eröffneten, um mit diesen die Bedürfnisse ihrer Landsleute zu befriedigen, änderten sich die Rahmenbedingungen sowie die Motivation zur Unternehmensgründung nach einigen Jahrzehnten. Im Fall der Tibeter in Delhi trifft dies bereits für die zweite Generation zu. Aus unterschiedlichen Gründen werden verschiedene Strategien verfolgt, um Kleinstunternehmen sowie kleine und mittlere Unternehmen aufzubauen.

Eine erste Strategie besteht darin, dass einige Tibeter, nachdem sie eine zunächst abhängige Beschäftigung gefunden haben, ihre Firma verlassen, obwohl sie dort für indische Verhältnisse gut verdient haben. Sie treibt das Bestreben nach Selbstständigkeit und Innovation:

„Once you are in a company you get salary every month, it gets higher also and your expense is more. So your saving is not so much, if you get 10.000 [Rupees] initially, later on you get 60.000 [Rupees] your expense is higher. So it's not much difference. So we want to come out of this rat race and do something new, especially in our community. " (eigenes Interview)

Hierfür können sie von Verwandten oft keine große finanzielle Unterstützung erwarten. Sie verfügen aber zum Teil über kleinere Ersparnisse. Dies bestätigen die erhobenen Daten, denn mehr als zwei Drittel der Befragten setzten ausschließlich ihr eigenes Kapital ein, knapp ein Viertel lieh sich Geld von Verwandten bzw. nahm einen Kredit bei Banken oder Freunden auf (Abb. 4). Die tibetischen Institutionen helfen eher durch Beratung als durch Finanzierungshilfe. Verwandtschafts- und Freundesnetzwerke bei gleichzeitig geringer finanzieller Unterstützung von außen, z. B. von indischen Banken, sind ein typisches Zeichen für diese ethnische Nischenökonomie.

Majnu Ka Tilla wählen die tibetischen Unternehmen als Standort wegen der dort dichten sozialen und ökonomischen Netzwerke. Für die Gründer eines Reisebüros etwa schien das Viertel optimal, da fast alle Reisewege der Exil-Tibeter in Indien über Delhi führen und Majnu Ka Tilla ein wichtiger Knotenpunkt vor allem für tibetische Handelsaktivitäten ist:

„All the Tibetans have to come to Majnu Ka Tilla, which is a very small place. It's a business place, a huge business place for the Tibetans. So we thought, let us first start from the Tibetan community, which we are confident of and then slowly we can go inside the Indian market. " (eigenes Interview)

Auch wenn Majnu Ka Tilla aus wirtschaftlichen Überlegungen als Standort innerhalb der tibetischen Migrantenökonomie gewählt wird, wird jedoch eine langfristige Ausweitung auf den indischen Markt von Beginn an mitgedacht. Zusätzlich führen einige Selbstständige Defizitanalysen durch, um zu ermitteln, welche Angebote in der Siedlung fehlen (beispielsweise wurde eine Laundry eröffnet, da sich die meisten Tibeter zwar eine Waschmaschine leisten können, es jedoch kaum Möglichkeiten im Viertel gibt, Wäsche zu trocknen): 


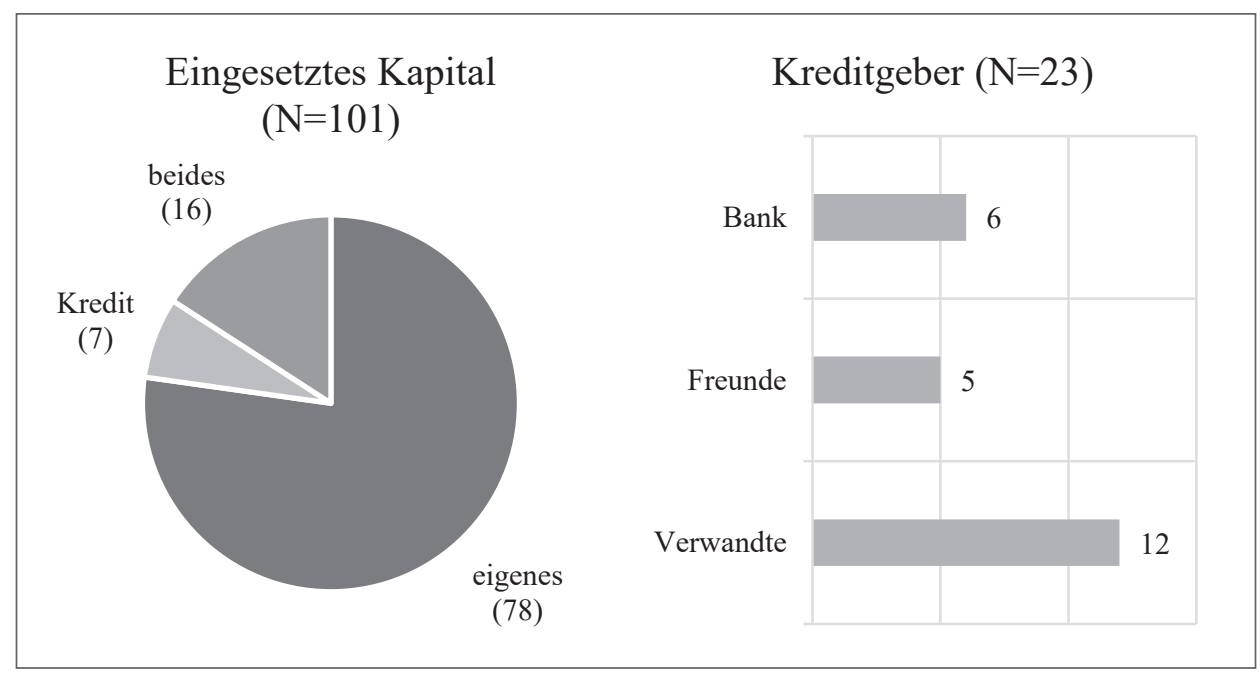

Quelle: Eigene Erhebung

Abb. 4: Eingesetztes Kapital und Kreditgeber

„And the one main thing is, here it's a very congested area, you don't have, even though people can afford to buy a washing machine at home, but then is no place, no place to dry clothes. " (eigenes Interview)

Um erfolgreich zu sein, werden neben kulturell angepassten Strategien für die tibetischen Kunden auch die im Viertel befindlichen Hotels und deren Klientel angesprochen.

Es wird zudem deutlich, dass sich die Rahmenbedingungen für die zweite Generation verändert haben. Aufgrund des zunächst eingenommenen Arbeitsplatzes in einem privaten Unternehmen verfügen die Tibeter dieser Generation über materielle und sozio-kulturelle Ressourcen. Sie suchen also nicht aus Not den Weg in die Selbstständigkeit, sondern aus dem Bestreben, eine größere Unabhängigkeit zu erlangen. Aufgrund ihrer Ausbildung (Schule/Universität) und der mehrjährigen Tätigkeit als Angestellte sowie auf Grundlage angeeigneten Fachwissens entwickeln sie ein ökonomisches Konzept zur Umsetzung ihrer Pläne. Sie verfügen über biographische und soziale Ressourcen, die der ersten Migrantengeneration fehlten. Diese suchte im Rahmen der „Flucht in die Selbstständigkeit“ eine Möglichkeit, mit geringem finanziellem, personellem und materiellem Aufwand den Landsleuten Produkte anzubieten, welche diese auf dem indischen Markt nicht finden konnten (Nischenökonomie), z. B. durch den Verkauf des tibetischen Biers Chang.

Auch die zweite Generation sucht eine ökonomische „Nische“, doch aus anderen Motiven und nicht ausschließlich für einen tibetischen Kundenstamm, sondern mit Orientierung auf fehlende Angebote im Viertel sowie mit modernem Marketing, das heißt, mit gezielter Werbung mit Blick auch auf Kunden anderer Nationalitäten. Majnu Ka Tilla wird dabei jedoch von ihnen noch bewusst als Standort gewählt, weil ihnen die ökonomischen Rahmenbedingungen des Viertels vertraut sind. 
Die Interaktion im Alltag zwischen Geschäftsleuten und Kunden, das heißt, auf intrapersonaler Ebene, zeigt dabei eine klare kulturelle Orientierung. Im Rahmen der kulturellen embeddedness ist hier der Informationsaustausch entscheidend. Der common cultural background liegt in der Übereinstimmung der Wünsche von Tibetern und Kunden anderer Nationalität. Gemeinsamer kultureller Hintergrund und face-to-face-Kontakte bilden dafür die Grundlage (AMIN und THRIFT 1997: „locally generated cultures“ der kulturellen „embeddedness“; PüTz 2004, S. 25). Die Chancen, welche die zweite Generation im Vergleich zur ersten Generation tibetischer Migranten besitzt, werden dabei klar reflektiert:

„, What we are happy is right now [...] everybody gets a good education, is educated, do something new. Not like you know like before our parents, they go for winter season only and then the rest of the year they just sit and do nothing. " (eigenes Interview)

Durch ihre höhere Bildung ist die Grundlage für die neuen Möglichkeiten der zweiten Generation gegeben, die dadurch nicht mehr allein die Selbstständigkeit als notgedrungene Überlebensstrategie wählen muss, sondern sich aus eigener Motivation - z. B. dem Wunsch nach Unabhängigkeit - für eine Unternehmensgründung entscheiden kann.

Bei einer zweiten Strategie verläuft der Weg in die Selbstständigkeit zunächst über staatliche Einrichtungen (Militär, Verwaltung), um sich im Gastland eine integrierte Position zu verschaffen.

„And after that then I took discharge from the [military] service and then did some tourism courses, because that was my interest and then I started business. And between also I did Dharma study for nearly three years in Buddhist Dialectic Institute in Tibetan Central Library. " (eigenes Interview)

Durch eine zusätzliche gezielte Aneignung von Kenntnissen, beispielsweise im Tourismussektor in Verbindung mit buddhistischen Studien, wird etwa eine Registrierung durch die indischen Behörden in Dharamsala, dem Sitz des Dalai Lama und der tibetischen Exilregierung, angestrebt. Tourismus, ausgerichtet auf ethnische Besonderheiten und ausländische Reisende, erlaubt eine breite Netzwerk- und Filialbildung:

,[W] operate tours, we operate adventure sports, we operate all this kind of transportation, we do all the ticket things, [...] a kind of event management, airport pick up, sightseeings [...] we do the pilgrimage tours, medicine tours, holiday tours and of course culture tours [...] in adventure sports, we do the trekking, we do the motorbiking tours, we do jeep safaris [...] we do the skiings and river rafting, river crossing, rock climbing [...] we take people to places and introduce the different cultures, like Hindu cultures or any other cultures, you know like Buddhist culture [...] we bring foreign students and then make exchange with the Tibetan students and schools [...] and we bring scholars and people to meet Lamas [...] and we organise conferences, and then the teachings, yoga and any therapies [...] and then language courses. " (eigenes Interview) 
Im Modell der Nischenökonomie zählt die Tourismusbranche gewissermaßen zur ,ersten Nische“" (Schaland 2009, S. 6). Die zweite Generation bedient sich dieser Nische und des Interesses für Tourismus und setzt auf die steigende Nachfrage, wobei gezielte Ausbildungswege verfolgt und biographische Ressourcen genützt werden. Diese locally generated cultures im Rahmen der kulturellen embeddedness dienen als Basis für ökonomisches und innovatives Handeln, welches durch Lernprozesse sowie Wissensaustausch bestimmt ist. Auch hier werden face-to-face-Kontakte nicht nur mit tibetischen Kunden, sondern auch mit Touristen anderer Nationalität hergestellt und eine strategische Transkulturalität praktiziert (PüTZ 2009, S. 78).

Dies geschieht im Einklang mit den indischen Behörden, das heißt, es erfolgt eine Registrierung des Unternehmens und Steuern werden abgeführt - im Unterschied zur ersten Generation, die sich weder registrieren ließ noch Steuern bezahlte. Mindestens ein knappes Drittel der Betriebe, vermutlich aber deutlich mehr (denn etwa ein Drittel der Interviewten verweigerte die Aussage), ist jedoch nicht registriert. Gut ein weiteres Drittel der Geschäfte ist zwar registriert, aber nicht notwendigerweise in der tatsächlich ausgeübten Branche, weil teils (z. B. für Reisebüros) hohe Kosten mit der „korrekten“ Registrierung verbunden sind.

Eine dritte Strategie tibetischer Selbstständiger verweist auf internationale Netzwerke. Mehrere Familienmitglieder sind mit ausländischen Partnern, z. B. Engländern und Japanern, verheiratet und leben im Ausland, etwa in London, Paris oder New York. Auch haben sie, beispielsweise über eine christliche Missionsschule, die von französischen und Schweizer Priestern geführt wurde, internationalen Anschluss erhalten, der für die Wahl eines Studiums und anschließende Arbeitsmöglichkeiten, teilweise zunächst im Ausland, mitbestimmend war. Die Verbindung nach Majnu Ka Tilla blieb über die Familienbeziehungen erhalten, womit der soziale Orientierungsraum gegeben war, der schließlich, teilweise durch Partnerwahl, teilweise durch Verwandtschafts- und Freundesnetzwerke, wieder eine „Rückkehrmigration“ erzeugte. Auch Bindungen zu eigenen ethnischen Organisationen spielen teilweise eine Rolle bei der Wahl des Investitionsraums (ein Interviewpartner war etwa Gründungsmitglied sowie Vorsitzender der Tibetan Chamber of Commerce):

„, So before we lost Tibet, my father was a business man in Tibet. So I guess I kind of inherited some of his business acronyms and trades. So besides of my architecture, which is predominately what I am doing, but besides that I am into publishing business and into constructions and in the hotel and guesthouse business, the catering business. Yes, I like to divert in all kind of places, where I think is business to be done. " (eigenes Interview)

Die beschriebenen Netzwerke spielen bei der Gründung von Unternehmen eine zentrale Rolle:

„I mean as far as the capital was concerned it wasn't so much of a problem, because my dad had some business in the northeast, so to start a business I hadn't really have to struggle for the initial capital. I managed to get that from my parents. But thereafter all the business in which I am right now, it's all started on my own. " (eigenes Interview) 
Die finanziellen Ressourcen der ersten Generation ermöglichen der zweiten Generation nicht nur eine bessere Ausbildung, sondern auch einen guten finanziellen Start in die Selbstständigkeit. Diese verfolgt gezielt eine strategische Transkulturalität: Lukrative Unternehmen (z. B. Architektenbüro, Bauunternehmen) werden nicht nur in Majnu Ka Tilla als Standort gegründet, sondern auch außerhalb, um vor allem auch Inder als Kunden zu gewinnen, denn die tibetische Community ist zu klein. Um erfolgreich geschäftliche Kontakte aufzubauen, sind regionale Sprachkenntnisse erforderlich (im Fall von Delhi: Hindi). Einige Interviewpartner betonten, dass indische Kunden am Telefon an ihrer Aussprache von Hindi nicht erkannten, dass sie Tibeter sind.

Bei der zukünftigen Unternehmensvergrößerung hoffen die Unternehmer, auf den indischen Markt expandieren zu können. Dies ist aber immer noch die Ausnahme (Abb. 5). Mehr als ein Drittel der Geschäftsinhaber kennt den eigenen Jahresumsatz nicht. Die Jahresumsätze eines weiteren Drittels (37 Unternehmen) liegen bei etwa einer Million Rupien, das heißt, etwa 14.000 Euro, somit im Subsistenzbereich; nur wenige erreichen mehr als 44.000 Euro (über 3 Millionen indische Rupien, INR).

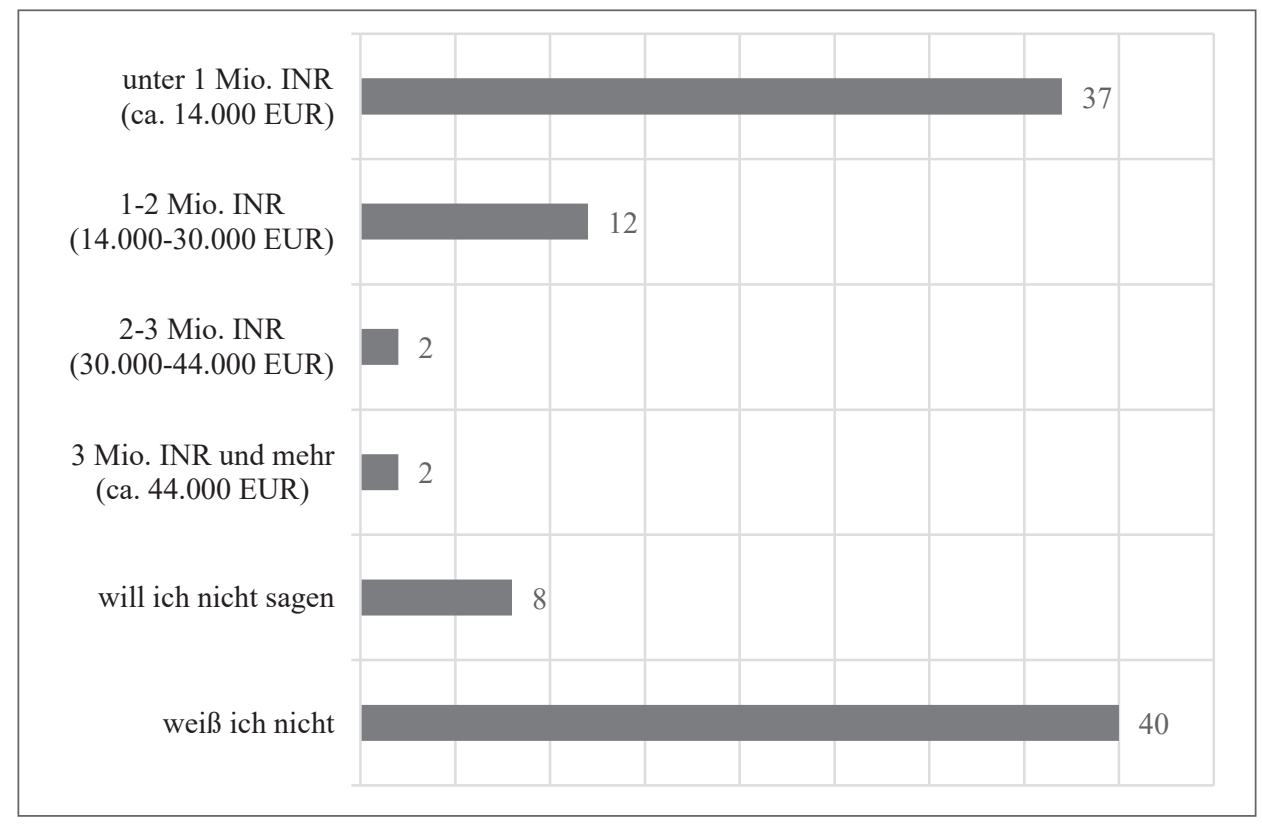

Quelle: Eigene Erhebung

Abb. 5: Jahresumsatz der tibetischen Selbstständigen in Majnu Ka Tilla (N=101)

In der Gemeinschaft von Majnu Ka Tilla engagieren sich die mittleren und großen Unternehmen geschäftlich in breiter Weise: im Verlagswesen oder im Gastronomie-, Hotel- und Baugewerbe. Je größer die Unternehmen werden, desto seltener werden jedoch Tibeter eingestellt, weil diese ihre Anstellung vor allem dazu nützen, um sich schnellst- 
möglich selbstständig zu machen oder nach Australien, Europa, Kanada oder in die USA zu emigrieren. Dies ist interessant, da sie nach einer ähnlichen Chance suchen, wie sie die Geschäftsinhaber hatten. Letztere aber wollen das Risiko eines schnellen Ausscheidens der jungen angestellten Tibeter nicht mehr eingehen.

Die drei Strategien zeigen auf, wie tibetische Migranten auf unterschiedliche Weise ökonomische Projekte umsetzen: Erstens agieren sie durch Einbringen von Arbeitserfahrung aus der Privatwirtschaft des Gastlandes, zweitens auf der Grundlage gesammelter Erfahrungen beim Militär und in der Verwaltung im Gastland, und drittens durch Nutzung vorhandener internationaler Netzwerke (wobei diese z. B. familiär basiert sind).

Durch die Legalisierung von Majnu Ka Tilla im Jahr 2012 besteht für die tibetische Migrantenökonomie eine größere Sicherheit. Seither werden in kleinerem oder größerem Umfang Investitionen in die Unternehmen ebenso wie in die privaten Häuser getätigt, auf die man vorher angesichts der Rechtsunsicherheit der Siedlung oft verzichtet hat.

Für den langfristigen Erhalt dieser Migrantenökonomie wird die junge tibetische Elite gebraucht. Eine Möglichkeit, sie in Indien zu halten, wäre, ihr Bemühen um die Erlangung der indischen Staatsangehörigkeit zu unterstützen. Mit den indischen Papieren kämen sie leichter an Kredite für Unternehmensgründungen und könnten Immobilien und Grundstücke kaufen. Ökonomische und kulturelle Faktoren befinden sich also in einem Spannungsverhältnis, wenngleich sich die Migrantenökonomie vordergründig über die Zugehörigkeit zur tibetischen Kultur definiert.

\section{Entwicklungspfade im tibetischen Viertel - Zukunft und Potenziale ökonomischer Entwicklung in Delhi}

Die Entwicklung des Viertels Majnu Ka Tilla von einem ,, ghetto of desperation, a jungle representing the elemental struggle for survival “ (SAMPHEL 2008, S. 3) zu einem angesehenen Geschäftsviertel zeigt, dass auch ursprünglich informelle Siedlungen - in Indien als „unauthorised colonies“ bezeichnet - erhebliche Entwicklungspotenziale aufweisen. Diese werden sowohl durch die Opportunitäten, in Form der Rahmenbedingungen des Gastlandes, bestimmt als auch durch die Ressourcen der Migranten (PüTz 2004, S. 16).

Mit den drei farbigen Eingangstoren zum Viertel, seinem Tempel, den bunten Auslagen sowie dem „exotischen Tibet-Flair“ wird Majnu Ka Tilla in Zukunft immer mehr zum „,urban heritage“ Delhis zählen - vergleichbar mit Singapurs „Kampong Glam“ (TANTOw 2012). Das könnte den Tourismus und damit die tibetische Migrantenökonomie des Viertels weiter fördern, so dass es auch in Zukunft das ,,commercial centre of the exile community, the hub of Tibetan commerce" (SAMPHEL 2006) in Indien bleibt.

\section{Literaturverzeichnis}

Amin A., Thrift N. (1997): Globalization, Socio-Economics, Territoriality. In: LeE R., Wills J. (Hrsg.): Geographies of Economies. London, John Wiley \& Sons, S. 147-157. 
Central Tibetan Administration (2012): His Holiness and Sikyong Grace Golden Jubilee of Tibetan Settlement in Delhi. - http://tibet.net/2012/12/27/his-holiness-andsikyong-grace-golden-jubilee-of-tibetan-settlement-in-delhi/ (Zugriff: August 2017).

FisChER-KRAPOHL I. (2010): Migrantenökonomie in der Stadt - Räumliche Verteilung, Potenziale und Good-Practice zur kommunalen Förderung. In: MATERn A., von Löwis S., Bruns A. (Hrsg.): Integration - Aktuelle Anforderungen und Strategien in der Stadt-, Raum- und Umweltplanung. Hannover, Akademie für Raumforschung und Landesplanung, S. 43-57 (= Arbeitsmaterial der Akademie für Raumforschung und Landesplanung 353).

Goldstein M. C. (1975): Tibetan Refugees in South India: A New Face to the Indo-Tibetan Interface. In: Tibet Society Bulletin, 9, S. 12-19.

Gupta I. (2011): Delhi's Mini Tibet: Majnu Ka Tilla. In: Hindustan Times, Ausgabe vom 18. Juni 2011. - http://www.hindustantimes.com/NewsFeed/Entertainment/LifeStyle/Article1710913.aspx (Zugriff: August 2016).

Hillmann F. (2001): Ethnische Ökonomien: eine Chance für die Städte und ihre Migrant(inn)en? In: Gestring N., Glasauer H., Hannemann C., Petrowsky W., Pohlan J. (Hrsg.): Jahrbuch StadtRegion 2001/02 (Schwerpunkt: Einwanderungsstadt). Opladen, Leske+Budrich, S. 35-56.

Kulinna M. (2007), Ethnomarketing in Deutschland. Die Konstruktion von Ethnizität durch Marketingakteure. Frankfurt/Main, Institut für Humangeographie der Universität Frankfurt (= Forum Humangeographie, 3).

Light I., Rosenstein C. (1995): Race, Ethnicity and Entrepreneurship in Urban America. New York, Aldine de Gruyter.

Methressel T. (1995): 35 Jahre Tibeter im Exil: Eine Analyse der sozio-ökonomischen Anpassungsprozesse in Indien und Nepal. Dissertation, Philipps-Universität Marburg, Deutschland.

Municipal Cooperation of Delhi (2012): Order for Unauthorized Colonies in Delhi. 04.09.2012. http://www.delhi.gov.in/wps/wcm/connect/093d2e00403d31c1b2fdb3879911cec1/9060001. $p d f ? M O D=A J P E R E S \& l m o d=-1380392877$ (Zugriff: März 2018).

PÜTZ R. (2003): Berliner Unternehmer türkischer Herkunft: „Ethnic“ Business? In: Die Erde. Zeitschrift der Gesellschaft für Erdkunde zu Berlin, 134, 3, S. 257-275.

PÜTZ, R. (2004): Transkulturalität als Praxis. Unternehmer türkischer Herkunft in Berlin. Bielefeld, Transkript.

PÜTZ, R. (2009): Perspektiven der „Transkulturalität als Praxis“: Unternehmer türkischer Herkunft in Berlin. In: Yildiz E., Mattausch B. (Hrsg.): Urban Recycling: Migration als Großstadt-Ressource. Basel, Birkhäuser, S. 63-81.

Resident Welfare Association (2010): Survey Plan of New Aruna Nagar Colony, 1:1000. New Delhi. SAmphel T. (2006): Majnu Ka Tilla Re-visited. - http://tibet.net/en/flash/2006/0906/12A0906.html (Zugriff: August 2016).

SAmphel T. (2008): Falling Through the Roof. New Delhi, Rupa \& Co.

SChaland A.-J. (2009): Selbstständige Migrantinnen und Migranten in wissensintensiven Dienstleistungsbranchen in Deutschland: Eine neue Nischenökonomie? Hamburg, Institut für Weltwirtschaft (= HWWI Research Papers, 3-18).

Tantow D. (2012): Politics of Heritage in Singapore: The Malay-Muslim Legacy of Kampong Glam. In: Indonesia and the Malay World, 40, 118, S. 332-353.

Tolciu A., Schaland A.-J. (2008): Selbstständige Migranten in Deutschland. In: Wirtschaftsdienst. Zeitschrift für Wirtschaftpolitik 2008, 8, S. 536-542. 